\title{
Impacto da Desinstitucionalização na Qualidade de Vida de Indivíduos com Transtornos Psiquiátricos
}

\author{
Impact of Deinstitutionalization on the Quality of Life of Individuals with Psychiatric \\ Disorders
}

\begin{abstract}
Impacto de la desinstitucionalización en la calidad de vida de las personas con trastornos psiquiátricos
\end{abstract}

Regina Lúcia Muniz de Almeidaa ${ }^{1}$, Heitor Motta Bini Pereira ${ }^{1 *}$, Samir Bernardo lle Mcauchar e Silva ${ }^{1}$, Camila de Oliveira Magno ${ }^{1}$, Ana Paula Ferreira ${ }^{1}$.

\section{RESUMO}

Objetivo. Caracterizar e analisar a qualidade de vida (QV) de usuários desinstitucionalizados atendidos pelo Centros de Atenção Psicossocial (CAPS) de uma cidade da Zona da Mata Mineira. Métodos. Trata-se de um estudo do tipo observacional, de corte transversal. Foram incluídos 75 participantes de ambos os sexos, com idade variando entre 18 e 65 anos, usuários do CAPS localizado em uma cidade da Zona da Mata Mineira. Todos os participantes foram submetidos inicialmente a uma anamnese contendo dados de histórico sócio demográfico e clínico e em seguida tiveram sua QV avaliada pelo instrumento validado SF-36 ${ }^{14}$. Resultados. Dos 75 pacientes entrevistados, 63 deles (84\%) informaram melhora significativa em sua QV e progresso no âmbito psicossocial após a desinstitucionalização e a entrada para o CAPS. Observou-se ainda que após a saída desses pacientes da internação hospitalar psiquiátrica prolongada, apenas $30(40 \%)$ retornaram ao mercado de trabalho e $5(6,6 \%)$ ao âmbito estudantil. Conclusão. Por fim, os dados deste trabalho reforçam a importância do processo de desinstitucionalização psiquiátrica na melhoria da QV dos usuários e familiares e na oferta da integralidade do cuidado, de modo que a valorização social, o desenvolvimento de potencialidades e a capacidade de atuar como agente ativo na sociedade sejam tão importantes quanto o acompanhamento clínico da enfermidade mental.

Palavras-chave: Desinstitucionalização, Qualidade de Vida, Psiquiatria.

\begin{abstract}
Objective To characterize and analyze the quality of life $(Q \circ L)$ of deinstitutionalized users served by the the Psychosocial Attention Center (CAPS) of a city in the Zona da Mata Mineira. Methods. This is an observational cross-sectional study. It was included 75 participants of both sexes, with ages varying between 18 and 65 years, users of the CAPS located in a city of the Zona da Mata Mineira. All participants were initially submitted to an anamnesis containing data from socio-demographic and clinical history and then had their QOL evaluated by the validated instrument SF-3614. Results. Of the 75 patients interviewed, 63 (84\%) reported significant improvement in their QOL and psychosocial progress after deinstitutionalization and entry to the CAPS. It was also observed that after the exit of these patients from prolonged psychiatric hospitalization, only $30(40 \%)$ returned to the labor market and $5(6.6 \%)$ returned to the student body. Conclusion. Finally, the data of this study reinforce the importance of the process of psychiatric deinstitutionalization in the improvement of the QoL of the users and relatives and in the offer of the integrality of the care, so that the social valorization, the development of potentialities and the capacity to act as an active agent in society are as important as the clinical follow-up of mental illness.
\end{abstract}

Key words: Deinstitutionalization, Quality of Life, Psychiatry.

${ }^{1}$ Faculdade de Ciências Médicas da Saúde de Juiz de Fora (SUPREMA), Juiz de Fora - Minas Gerais.

*E-mail: heitorbini3@gmail.com 


\section{RESUMEN}

Objetivo Caracterizar y analizar la calidad de vida (QoL) de los usuarios desinstitucionalizados atendidos por los Centro de Atención Psicosocial (CAPS) de una ciudad en la Zona da Mata Mineira. Los metodos Este es un estudio observacional, transversal. Se incluyeron 75 participantes de ambos sexos, con edades entre los 18 y 65 años, usuarios del CAPS ubicado en una ciudad de la Zona da Mata Mineira. Inicialmente, todos los participantes fueron sometidos a una anamnesis que contenía datos de la historia sociodemográfica y clínica y luego se evaluó su QOL mediante el instrumento validado SF-3614. Resultados De los 75 pacientes entrevistados, 63 (84\%) informaron una mejoría significativa en su calidad de vida y progreso psicosocial después de la desinstitucionalización y el ingreso a los CAPS. También se observó que después de la salida de estos pacientes de una hospitalización psiquiátrica prolongada, solo $30(40 \%)$ regresaron al mercado laboral y $5(6,6 \%)$ regresaron al cuerpo estudiantil. Conclusión Finalmente, los datos de este estudio refuerzan la importancia del proceso de desinstitucionalización psiquiátrica en la mejora de la calidad de vida de los usuarios y familiares y en la oferta de la integralidad de la atención, de modo que la valorización social, el desarrollo de potencialidades y la capacidad de actuar como agente activo. En la sociedad son tan importantes como el seguimiento clínico de la enfermedad mental.

Palabras clave: Desinstitucionalización, Calidad de Vida, Psiquiatría.

\section{INTRODUÇÃO}

O processo de desinstitucionalização da assistência psiquiátrica no Brasil iniciou-se a partir da década de 1990, com o objetivo de diminuir as internações psiquiátricas e inserir os pacientes na sociedade (GONÇALVES AM e SENA RR, 2001).

Esse movimento deveu-se ao fato de que o espaço fechado do hospital psiquiátrico contribui para efeitos deletérios associados à diminuição na qualidade de vida $(\mathrm{QV})$, perda da liberdade e dos valores culturais (HIRDES A, 2009).

Nesse sentido é possível apontar que a desinstitucionalização e a reinserção social dos pacientes são fundamentais para melhorias na QV Khoury HTT e Sá-Neves AC (2014); Kunitoh N (2013) de pessoas que passaram pelo processo de internação psiquiátrica.

A QV, é definida pela Organização Mundial da Saúde (OMS) como a "percepção do indivíduo de sua posição na vida, no contexto da cultura e sistema de valores nos quais ele vive, e em relação aos seus objetivos, expectativas, padrões e preocupações" (KHOURY HTT e SÁ-NEVES AC, 2014);

Destaca-se como desafio existente para a reintegração desses pacientes na comunidade, a transferência dos cuidados hospitalares para alternativas de atendimento externo Alverga AR (2006); Fagundes Júnior HM et al. (2016), bem como a reorganização na Rede de Atenção à Saúde Mental no Brasil.

Esse processo teve início a partir da criação de CAPS, Serviços de Residências Terapêuticas; o Programa de Volta para Casa e centros de convivência (MINISTÉRIO DA SAÚDE, 2004; AMORIM AKMA e DIMENSTEIN M, 2009).

O movimento de Reforma Psiquiátrica possibilitou significativo avanço na Política de Saúde Mental, sobretudo no reconhecimento dos direitos dos pacientes e no retorno destes ao âmbito familiar e da comunidade, transpondo o paradigma de que o tratamento a este público deveria ser realizado somente através de internações hospitalares (SILVA EKB e ROSA LCS, 2014).

Algumas evidências científicas Braun P e Kochansky G (1981); Avison WR e Speechley KN (1987) mostraram que a desinstitucionalização psiquiátrica proporcionou melhora importante na estabilidade do estado psicológico e aumento no desempenho social, além de promover melhora das habilidades sociais, interpessoais e de trabalho.

Ciente dessas evidências, o governo brasileiro, na atualidade, assegura o seu auxílio através dos CAPS, que atuam por uma ferramenta chamada Projeto de Terapia Única, que possui como diretriz a recuperação 
da autonomia do paciente e a realização de oficinas e espaços culturais, fundamentais para a QV e para que haja reintegração em um âmbito coletivo (PINHO ES, et al., 2012).

Além disso, os usuários desse sistema possuem apoio governamental garantido pela Lei Federal 10.708 que dispõe, através do Programa de Volta para Casa, um auxílio-reabilitação psicossocial e econômico no valor de 240 reais mensais, que, mesmo sendo pequeno, contribui no sentido de estímulo à autonomia, melhora na QV e reinserção social adequada (MINISTÉRIO DA SAÚDE, 2018).

Face ao exposto e sabendo da importância do processo de desinstitucionalização do paciente psiquiátrico com a sua reintegração na comunidade, o objetivo do presente estudo foi caracterizar e analisar a QV de usuários desinstitucionalizados atendidos pelo CAPS de uma cidade da Zona da Mata Mineira.

\section{MÉTODOS}

O estudo é do tipo observacional, de corte transversal. Foram selecionados 75 participantes através de amostragem por conveniência, de ambos os sexos, com idade variando entre 18 e 65 anos, usuários do Centro de Atenção Psicossocial (CAPS) localizado em uma cidade da Zona da Mata Mineira.

Todos os participantes foram submetidos inicialmente a uma anamnese contendo dados de histórico sóciodemográfico e clínico. Em seguida tiveram sua QV avaliada pelo instrumento validado Short-Form Health Survey (SF-36) Ciconelli RM, et al., (1999) aplicado por um único avaliador no mesmo dia.

O SF-36 segundo Ciconelli RM, et al., (1999) é um instrumento validado no Brasil utilizado amplamente em diversas populações com objetivo de avaliar a QV considerando os domínios capacidade funcional, limitação por aspectos físicos, dor, estado geral de saúde, vitalidade, aspectos sociais, aspectos emocionais e saúde mental.

Cabe destacar ainda que os dados pessoais coletados foram catalogados em uma ficha de avaliação individual garantido sigilo e integridade ética dos avaliados. Para tratamento dos dados, foi utilizada estatística descritiva sendo a (média \pm DP) calculada para variáveis paramétricas.

O teste de Shapiro Wilk foi aplicado para testar a normalidade dos dados. Para análise, foi utilizado o software estatístico GraphPad Prism 6.0 (GraphPad Software Inc., San Diego, CA, EUA).

Ressalta-se que o presente estudo foi aprovado pelo Comitê de Ética em Pesquisa da Faculdade de Ciências Médicas e da Saúde de Juiz de Fora - SUPREMA, sob parecer número 2.931.661.

\section{RESULTADOS}

Observou-se na amostra investigada uma classe predominantemente ativa em questões produtivas e trabalhistas. Com a saída desses pacientes da internação hospitalar psiquiátrica prolongada, apenas 30 (40\%) retornaram ao mercado de trabalho e $5(6,6 \%)$ ao âmbito estudantil.

Os achados mostram ainda que 19 pacientes (25,3\%) eram aposentados por invalidez pelo Instituto Nacional de Seguro Social (INSS) em consequência da doença mental.

Observa-se ainda que a enfermidade mais prevalente desses sujeitos era a depressão (presente em $44 \%$ dos participantes).

Considerando o fato de que os entrevistados estão inseridos na faixa etária dos 18 aos 65 anos, população potencialmente ativa do ponto de vista econômico segundo o Instituto Brasileiro de Geografia e Estatística (2019), verifica-se o caráter incapacitante dos transtornos psiquiátricos.

Todas as questões do SF-36 foram respondidas. O tempo médio de aplicação do SF-36 foi de 20 minutos. As características sociodemográficas e clínicas da amostra estão apresentadas na Tabela 1. 
Tabela 1 - Características sociodemográficas e clínicas e da amostra.

\begin{tabular}{|c|c|c|}
\hline & $n=75$ & $(\%)$ \\
\hline \multicolumn{3}{|l|}{ Sexo } \\
\hline Feminino & 35 & 46,6 \\
\hline Masculino & 40 & 53,3 \\
\hline \multicolumn{3}{|l|}{ Grau de Escolaridade } \\
\hline Alfabetizados & 62 & 82,6 \\
\hline Não - Alfabetizados & 13 & 17,3 \\
\hline \multicolumn{3}{|l|}{ Comorbidades } \\
\hline Hipertensão Arterial & 22 & 29,3 \\
\hline Diabetes & 9 & 12 \\
\hline Outras & 3 & 4 \\
\hline Nenhuma & 41 & 54,6 \\
\hline \multicolumn{3}{|l|}{ Ocupação } \\
\hline Aposentados pelo INSS (consequência da doença) & 19 & 25,3 \\
\hline Trabalhador autônomo & 30 & 40 \\
\hline Não Trabalham & 21 & 28 \\
\hline Estudantes & 5 & 6,6 \\
\hline \multicolumn{3}{|l|}{ Diagnóstico Clínico } \\
\hline Dependência Química & 10 & 13,30 \\
\hline Transtorno Bipolar & 15 & 20 \\
\hline Esquizofrenia & 17 & 22,60 \\
\hline Depressão & 33 & 44 \\
\hline \multicolumn{3}{|l|}{ Deficiências/Limitações } \\
\hline Distúrbios da fala & 14 & 18,6 \\
\hline Coordenação motora comprometida & 9 & 12 \\
\hline Déficit cognitivo & 11 & 14,6 \\
\hline Ausência & 41 & 54,6 \\
\hline
\end{tabular}

Fonte: Almeida RLM, Pereira HMB, Silva SBLM, et al., 2019.

O questionário SF-36 teve seus domínios calculados e agrupados separadamente. Os valores médios do SF-36 estão apresentados na Tabela 2.

Tabela 2 - Valores obtidos para cada componente do questionário SF-36.

\begin{tabular}{lcc}
\multicolumn{1}{c}{ Componentes } & Média (DP) & Mínimo - Máximo \\
\hline Capacidade Funcional & $47,8(30,2)$ & $10-100$ \\
Aspectos Físicos & $82,6(31,3)$ & $0-100$ \\
Dor & $48,2(21,2)$ & $0-100$ \\
Estado Geral da Saúde & $49,2(16,4)$ & $5,7-85$ \\
Vitalidade & $56,4(20,3)$ & $0-100$ \\
Aspectos gerais & $56,9(19,3)$ & $13,3-100$ \\
Aspectos Emocionais & $54,9(39,4)$ & $0-100$ \\
Saúde Mental & $54,1(21,1)$ & $13-100$ \\
\hline
\end{tabular}

Fonte: Almeida RLM, Pereira HMB, Silva SBLM, et al., 2019.

\section{DISCUSSÃO}

A desinstitucionalização da assistência psiquiátrica no Brasil está incluída no novo modelo de atenção em saúde mental, cujo objetivo é promover maior QV aos indivíduos com transtornos mentais e aos seus familiares (DIMENSTEIN M, 2006; BOSI MLM, et al., 2016). Nesse contexto, os CAPS se configuram como um dos principais serviços estratégicos substitutivos à internação hospitalar psiquiátrica prolongada. As modalidades de atendimento no CAPS compõem um universo de prática que visa dar suporte ao paciente em crise, envolvendo o tratamento clínico, a compreensão dos aspectos biopsicossociais, a humanização e integralidade do cuidado, com intervenções que visam reinseri-lo no contexto social e familiar. O CAPS é composto por uma equipe multidisciplinar, a saber: psiquiatras, assistentes sociais, psicólogos, terapeutas ocupacionais, enfermeiros, auxiliares de enfermagem e monitores sociais. 
Por ser um movimento relativamente recente na história brasileira (marcada a partir da década de 90), torna-se relevante para a comunidade científica e para as entidades públicas a análise dos efeitos a longo prazo da desinstitucionalização na QV dos pacientes com desordens psicoafetivas (HIRDES A, 2009; LUCENA MAS e BEZERRA AFB, 2012; SILVA PRF, et al., 2017). Dos 75 pacientes entrevistados, 63 deles (84\%) informaram melhora significativa em sua QV e progresso no âmbito psicossocial após a desinstitucionalização e entrada para o CAPS III. Em contrapartida, 12 participantes (16\%) afirmaram não terem obtido benefício significativo em sua QV após a entrada ao CAPS.

Observou-se que a maioria dos entrevistados que apontaram não haver melhora após a saída da internação hospitalar psiquiátrica prolongada e adesão aos serviços oferecidos pelo CAPS compunham os pacientes com sequelas clínicas, sendo a esquizofrenia e a dependência química os diagnósticos prevalentes encontrados nesse grupo, uma vez que esses pacientes não possuíam autonomia necessária para seus cuidados pessoais. Contudo, o resultado obtido revela um impacto positivo do programa de saúde do governo CAPS na grande maioria dos usuários.

Os resultados do presente estudo corroboram os achados de Botelho JV e Lima MV (2015), que objetivaram analisar a integração social de usuários de um CAPS da cidade de Vitória da Conquista, Bahia, através de um relato de experiência tendo como base as percepções e emoções dos pacientes com relação aos serviços prestados pela instituição. Através de uma observação anônima norteada pelos fatores emoção, sociabilidade, apoio e valorização social, os pesquisadores observaram satisfação dos usuários no que tange aos quatro itens mencionados, bem como a preocupação dos profissionais em promover um ambiente que estimule os usuários a desenvolver sua autonomia e participação na sociedade. O estudo afirma ainda que a experiência relatada sugere que há melhoria na qualidade de vida dos participantes do referido CAPS.

Apesar dos evidentes benefícios do novo modelo de atenção em saúde mental, cabe ressaltar que o processo de desinstitucionalização possui relevantes obstáculos para sua plena implantação. Salles MM e Barros (2013), realizaram um estudo qualitativo com objetivo de identificar e analisar a percepção de usuários e pessoas da sua rede social sobre doença mental e inclusão social, com 29 participantes ao todo (17 usuários e 12 pessoas de seu convívio social). Os resultados evidenciaram que os entrevistados compreendem a inclusão social como um conjunto dinâmico de aspectos envolvendo trabalho, educação, renda, poder contratual e ser aceito na sua diferença. Ainda que a percepção tenha sido positiva, os participantes relataram dificuldades de inclusão social devido a preconceitos e discriminações e à própria herança cultural negativa sobre pacientes com transtornos psiquiátricos não poderem conviver pacificamente em sociedade.

Nesse mesmo contexto, Santos JC, et al., (2016) demonstraram que, da perspectiva dos trabalhadores de três CAPS na cidade de São Paulo, o estigma cultural é um importante entrave à reinserção biopsicossocial dos usuários. Os estudos apontam para o que acreditamos ser o maior desafio da reforma psiquiátrica brasileira: a inclusão social do paciente com doença mental.

Desta forma, os achados do presente estudo sugerem melhoria importante da QV na maioria dos usuários após o advento da desinstitucionalização. Tal fato, é de extrema relevância para reinserção deste indivíduo na comunidade, o que endossa a importância do novo modelo de atenção na promoção da saúde global do paciente psiquiátrico e seus familiares, considerando os aspectos biopsicossociais e a integralidade do indivíduo.

\section{CONCLUSÃO}

Por fim, os dados deste trabalho reforçam a importância do processo de desinstitucionalização psiquiátrica na melhoria da QV dos usuários e familiares e na oferta da integralidade do cuidado, de modo que a valorização social, o desenvolvimento de potencialidades e a capacidade de atuar como agente ativo na sociedade, sejam tão importantes quanto o acompanhamento clínico da enfermidade mental. Mesmo com uma legislação efetiva sobre os transtornos mentais, a qualificação de equipes multiprofissionais para atendimento de pacientes com distúrbios psiquiátricos é necessária para a mudança de paradigma sobre a doença mental. 


\section{REFERÊNCIAS}

1. GONÇALVES AM, SENA RR. A Reforma Psiquiátrica no Brasil: contextualização e reflexos sobre o cuidado com o doente mental na família. Rev. Latino-Americano, 2001; 9(2): 48-55.

2. HIRDES A. A reforma psiquiátrica no Brasil: uma (re)visão. Ciência \& Saúde Coletiva, 2009; 14(1): $297-305$.

3. KHOURY HTT, SÁ-NEVES AC. Percepção de controle e qualidade de vida: comparação entre idosos institucionalizados e não institucionalizados. Rev. Bras. Geriatr. Gerontol., 2014; 17(3): 553-565.

4. KUNITOH N. From hospital to the community: th e influence of deinstitutionalization on discharged long-stay psychiatric patients. Psychiatry Clin Neurosci, 2013; 67(6): 384-396.

5. ALVERGA AR, DIMENSTEIN M. A reforma psiquiátrica e os desafios na desinstitucionalização da loucura. Interface (Botucatu), 2006; 10(20): 299-316.

6. FAGUNDES JÚNIOR HM, DESVIAT M, SILVA PRF. Reforma Psiquiátrica no Rio de Janeiro: situação atual e perspectivas futuras. Ciência \& Saúde Coletiva, 2016; 21(5): 1449-1460.

7. Ministério da Saúde. Secretaria de Atenção à Saúde. Departamento de Ações Programáticas Estratégicas. Saúde Mental no SUS: os Centros de Atenção Psicossocial. Brasília: Ministério da Saúde, 2004.

8. AMORIM AKMA, DIMENSTEIN M. Desinstitucionalização em saúde mental e práticas de cuidado no contexto do serviço residencial terapêutico. Ciência \& Saúde Coletiva, 2009; 14(1): 195-204.

9. SILVA, EKB, ROSA LCS. Desinstitucionalização psiquiátrica no Brasil: riscos de desresponsibilização do Estado. R Katál, 2014; 17(2): 252-260.

10. BRAUN P, KOCHANSKY G, SHAPIRO R et al. Overview: deinstitutionalization of psychiatric patients, a critical review of outcome studies. Am J Psychiatry, 1981; 138(6): 736-749.

11. AVISON WR, SPEECHLEY KN. The discharged psychiatric patient: A review of social, social-psychological, and psychiatric correlates of outcome. Am J Psychiatry, 1987; 144(1): 10-18.

12. PINHO ES, SOUZA ACS, ESPERIDIÃO E. Working Processes of professionals at Psychosocial Care Centers (CAPS): an integrative review. Ciência \& Saúde Coletiva, 2018; 23(1): 141-152.

13. Ministério da Saúde. Biblioteca Virtual da Saúde. Manual do Programa De Volta Para Casa. 2018.

14. CICONELLI RM, FERRAZ MB, SANTOS $W$ et al. Tradução para a língua portuguesa e validação do questionário genérico de avaliação de qualidade de vida SF-36 (Brasil SF-36). Rev. Bras. Reumatol., 1999; 39: 143-150.

15. DIMENSTEIN M. O desafio da política de saúde mental: a (re)inserção dos portadores de transtornos mentais. Mental, 2006; 4(6): 69-83.

16. BOSI MLM, MELO AKS, CARVALHO LB et al. Determinantes sociais em saúde (mental): analisando uma experiência não governamental sob a ótica de atores implicados. Rev Bras Epidemiol, 2016; 17(2): 126-35.

17. Brasil. Portaria/GM № 336, de 19 de fevereiro de 2002. Portaria que define e estabelece diretrizes para o funcionamento dos Centros de Atenção Psicossocial. Estes serviços passam a ser categorizados por porte e clientela, recebendo as denominações de CAPS I, CAPS II, CAPS III, CAPSi e CAPSad. Documento fundamental para gestores e trabalhadores em saúde mental. Diário Oficial da União 2001; 20 fev.

18. PINHO ES, SOUZA ACS, ESPERIDIÃO E. Processos de trabalho dos profissionais dos Centros de Atenção Psicossocial: revisão integrativa. Ciência \& Saúde Coletiva, 2018; 23(1): 141-51.

19. LUCENA MAS, BEZERRA AFB. Reflexões sobre a gestão de processos de desinstitucionalização. Ciência \& Saúde Coletiva, 2012; 17(9): 2447-2456.

20. SILVA PRF, CARVALHO MCA, CAVALCANI MT et al. Desinstitucionalização de pacientes de longa permanência de um hospital psiquiátrico no Rio de Janeiro. Ciência \& Saúde Coletiva, 2017; 22(7): 2341-2352.

21. Indicadores sociais mínimos. Conceitos. In: Instituto Brasileiro de Geografia e Estatística. Disponível em: https://www.ibge.gov.br/home/estatistica/populacao/condicaodevida/indicadoresminimos;conceitos.shtm. Acesso em: 29 Mar. 2019.

22. BOTELHO JV, LIMA MV. Percepção das emoções dos usuários do CAPS II: um relato de experiência. Fractal, Rev. Psicol., 2015; 27(2): 160-164.

23. SALLES MM. BARROS S. Representações sociais de usuários de um Centro de Atenção Psicossocial e pessoas de sua rede sobre doença mental e inclusão social. Saude Soc., 2013; 22(4): 1059-1071.

24. SANTOS JC, BARROS S, SANTOS IMM. Stigma: The Perspective of Workers on Community Mental Health ServicesBrazil. Glob Qual Nurs Res, 2016; 3: 1-9. 